\title{
Overcoming obstacles: new management options
}

\author{
L Heinemann \\ Profil Institute for Metabolic Research GmbH, Hellersbergstr. 9, D-41460 Neuss, Germany \\ (Correspondence should be addressed to L Heinemann; Email: Lutz.Heinemann@profil-research.de)
}

\begin{abstract}
The outlook for patients with type 2 diabetes looks set to improve with the availability of new diabetes management options that provide more comprehensive control of blood glucose levels and/or encourage better patient compliance than previous alternatives. New insulin analogues, such as insulin lispro, aspart and glargine, allow more physiological insulin replacement and greater freedom in the timing and content of meals, compared with regular insulin preparations. The development of novel non-invasive routes of insulin administration promises to further improve diabetes management. Many barriers to initiating insulin relate to the need for frequent insulin injections, fears that insulin injections will be painful and difficult to administer, and concerns about hypoglycaemia and weight gain. Thus, each measure that reduces these barriers will help to prevent inappropriate delays in starting insulin therapy as well as to promote better compliance with therapy. The output from continuous glucose monitoring devices will assist accurate insulin replacement, which is difficult using point-estimates of blood glucose. Such devices will hopefully also circumvent the need for finger stick tests. There are several novel therapies in development that will further expand the portfolio of treatment options for patients with type 2 diabetes. Improved quality and choice of diabetes management options will provide doctors with the tools they require to develop tailored treatment plans, increase the probability that treatment goals are achieved and thereby reduce the risk of patients developing late-stage diabetes-related complications.
\end{abstract}

European Journal of Endocrinology 151 T23-T27

\section{Introduction}

Advances in drug therapy and diabetes technology are enabling more flexible and convenient diabetes management, with improved prandial and basal metabolic control. For many years, patients with type 2 diabetes taking insulin have been required to adjust their diet, mealtimes and exercise patterns to fit in with their treatment regimen. Patients have been restricted from eating meals rich in rapidly absorbable carbohydrates to avoid post-prandial hyperglycaemia that cannot be adequately controlled using subcutaneous injections of soluble (or regular) insulin. Such restrictions make it difficult for patients to live a 'normal' life and encourage non-compliance with therapy. However, the development of rapid-acting insulin analogues that offer improved prandial blood glucose regulation with greater flexibility means that therapy can be adjusted according to the timing and carbohydrate content of meals.

Prospective intervention studies such as the Diabetes Control and Complications Trial (DCCT) and the UK Prospective Diabetes Study (UKPDS) have demonstrated the benefits of intensive insulin therapy (designed to produce near-normoglycaemia) in preventing the development of long-term vascular complications. Increasingly, stringent blood glucose targets have made diabetes management more challenging for both patients and physicians, and they demand better management options. This mini-review summarises some of the drugs and devices that have been developed and are in development in response to this growing need for effective diabetes therapies. These new management options - optimised insulin formulations, glucose monitoring devices, novel routes of insulin administration and novel pharmacological agents - should help to improve diabetes outcomes by overcoming obstacles to compliance and thereby improving glycaemic control.

\section{Optimised insulin therapy}

The principal idea of intensive insulin therapy is to mimic insulin secretion by the healthy pancreas. Therefore, intensive insulin treatment regimens usually involve pre-prandial bolus injection of short-acting insulin (to control meal-related glycaemia) together with injection of intermediate- or long-acting insulin (to control between-meal and nocturnal glycaemia). However, the pharmacokinetic and pharmacodynamic properties of regular human insulin preparations hamper physiological insulin replacement. Regular human insulin is absorbed into the bloodstream slowly and with poor reproducibility when it is injected into subcutaneous tissue. Injections of regular insulin should therefore be 
made 30 min before meals (although recommendations vary considerably), which can lead to pre-prandial hypoglycaemia if the meal is missed or delayed. Moreover, prandial hypoinsulinaemia and postprandial hyperinsulinaemia may occur because of the slow onset, but prolonged duration, of action of regular insulin.

Recently, insulin analogues with more physiological time-action profiles than conventional insulin preparations have been developed. Substituting one or several amino acids of the primary structure of the human insulin molecule results in insulin analogues with different physico-chemical, biological and pharmacodynamic properties compared with regular insulin. Rapid-acting analogues, such as insulin lispro and insulin aspart, have a more rapid onset of action and a shorter duration of action than regular insulin after subcutaneous injection owing to amino acid substitutions that reduce the association of insulin monomers into hexamers (thus enabling more rapid absorption into the bloodstream). As well as improving postprandial blood glucose regulation, this gives patients more flexibility by allowing insulin to be injected immediately before meals. Some studies show that rapid-acting insulin analogues can reduce haemoglobin $(\mathrm{Hb}) \mathrm{A}_{1 \mathrm{c}}$ a further $0.1-0.3 \%$ compared with regular insulin, provided that basal insulin replacement is adjusted appropriately. There is also evidence that the use of rapid-acting insulin analogues is associated with fewer hypoglycaemic events compared with regular insulin, but the reduction in incidence is not substantial in the majority of published studies (reviewed in 1,2).

Long-acting insulin analogues, e.g. insulin glargine and insulin detemir, have been developed by changing the structure of the insulin molecule to retard its absorption into the bloodstream. Subcutaneous injection of long-acting insulin analogues induce a more constant time-action profile and a longer duration of action (lasting at least $24 \mathrm{~h}$ for insulin glargine) compared with conventional long-acting insulin formulations, which have pronounced peaks and a shorter duration of action $(3-8)$. They have a comparable or superior effect on $\mathrm{HbA}_{1 \mathrm{c}}$, and also tend to reduce the risk of hypoglycaemic events $(2,4-8)$.

Most patients with type 2 diabetes eventually require insulin because of declining beta-cell function that cannot be prevented or overcome by use of oral agents. With the availability of several different classes of oral agents and the potential of multiple different mono- and combination-therapy treatment regimens, insulin has become widely considered as a treatment of last resort in patients with type 2 diabetes. However, the benefits of initiating insulin therapy earlier in the management of type 2 diabetes are receiving increasing attention (9). Unlike oral agents, insulin can achieve effective glycaemic control and maintain this in the long-term. Insulin also has the longest established safety profile of all the glucose-lowering agents available on the market.
Despite these favourable therapeutic characteristics, both patients and physicians are often reluctant to begin insulin therapy. Many of the barriers to initiating insulin therapy relate to the inconvenience of insulin injections, worries about proper injection technique, needle-phobia and concerns about hypoglycaemia, deterioration in health, and weight gain. These issues can also discourage compliance with therapy among patients who are prescribed insulin. The availability of new insulin formulations and more convenient, noninvasive routes of insulin delivery promise to help overcome these real and perceived obstacles to insulin, thereby improving patient acceptance and encouraging its use earlier in the treatment process.

\section{Alternative routes of insulin administration}

Advanced injection devices, such as insulin pens, have been designed in an attempt to minimise needle phobia and overcome the inconvenience issues associated with conventional subcutaneous insulin injections. However, recent technological advances have allowed needle-free options to be considered as realistic alternatives. Several novel non-invasive routes of insulin delivery are being investigated using transdermal, intra-nasal, oral enteric, oral buccal and pulmonary delivery routes. Pulmonary delivery is the most promising non-subcutaneous route of insulin administration investigated to date. Inhaled insulin would allow patients with type 2 diabetes to intensify their insulin therapy and to improve their glycaemic control with fewer or no insulin injections. Several companies are currently developing inhaled insulin systems; Exubera (being developed by Pfizer Inc. (New York, NY, USA) and Aventis Pharma (Bridgewater, NJ, USA) in conjunction with Nektar Therapeutics (San Carlos, CA, USA)) is the most advanced product in terms of clinical development.

Inhaled insulin is absorbed quickly into the systemic bloodstream enabling a rapid onset of action. Clinicalexperimental trials have established that inhaled insulin has a reproducible time-action profile (10) with a faster onset of action than that seen after subcutaneous injection of regular human insulin (11) and one which is similar to subcutaneous injection of insulin lispro (Fig. 1). Its duration of action is intermediate between subcutaneous regular insulin and insulin lispro (11). Inhaled insulin has been observed to lower prandial glucose levels more effectively than subcutaneous regular insulin, without increasing the risk of hypoglycaemic events. In addition, studies have shown that inhaled insulin provides greater overall patient satisfaction, convenience and ease of use compared with subcutaneous insulin, for up to 1 year, in patients with type 1 or type 2 diabetes (12-14). These improvements in patient satisfaction correlate with improvements in glycaemic control, which, however, was comparable with inhaled and subcutaneous insulin (14). 


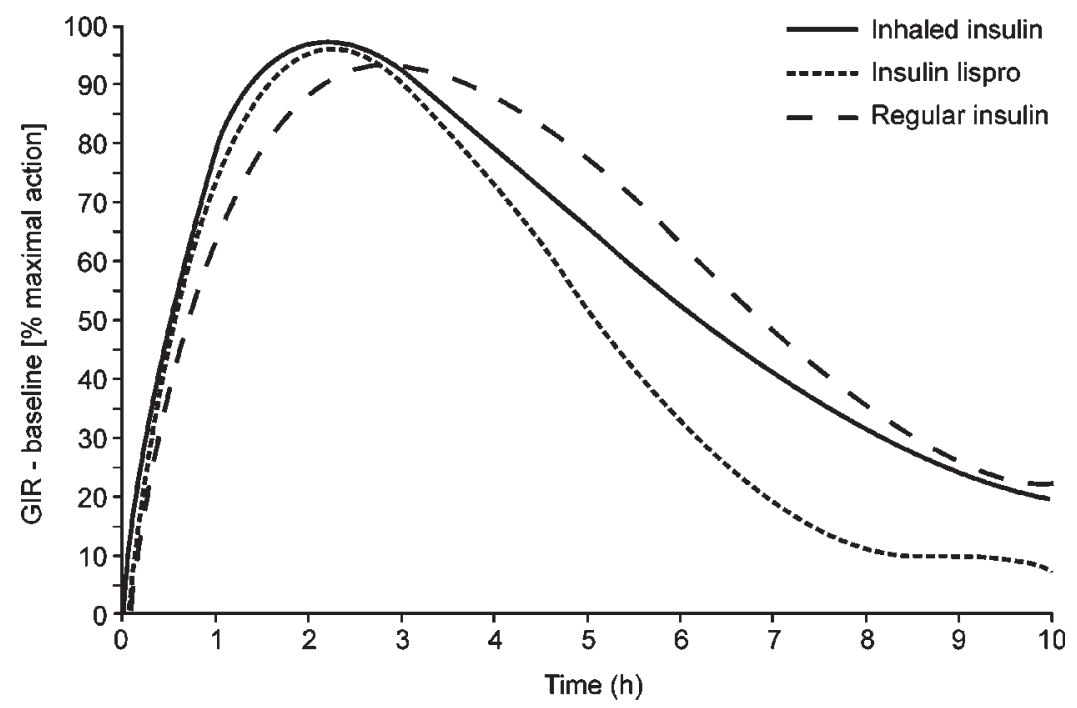

Figure 1 Time-action profiles of inhaled insulin (6 mg), subcutaneous regular insulin (18IU) and subcutaneous insulin lispro (18IU; $n=18$ healthy volunteers). GIR, glucose infusion rate. (Reprinted with permission from the American Diabetes Association (11).)

In Phase 2 and Phase 3 trials with inhaled insulin a considerable increase in insulin antibody levels was observed, especially in patients with type 1 diabetes. Also, a small but significant reduction in carbon monoxide diffusion capacity and forced lung volume in $1 \mathrm{~s}$ was observed. However, in further studies specifically designed to characterise these changes, the initially observed small declines in lung function do not progress further and appear to be reversible and the appearance of insulin binding antibodies appears not to be correlated to indices of metabolic control and clinical safety.

\section{Glucose monitoring}

Whereas the normal pancreas continuously adjusts the amount of insulin it secretes depending on the prevailing blood glucose concentration, patients undergoing intensive insulin therapy are advised to monitor their blood glucose levels at least 3-4 times a day and adjust their bolus insulin dose. However, spot-measurements do not give an accurate impression of the true $24 \mathrm{~h}$ glucose profile and cannot indicate the direction or trend of changes in blood glucose levels. There may be very large excursions in blood glucose levels, especially in the postprandial period, which cannot be detected by pre-prandial glucose measurements alone. Additionally, night-time glycaemia cannot be practically measured by self-monitoring blood glucose. The need for frequent blood glucose monitoring requiring fingerstick tests also adds to the daily burden of managing diabetes. Many patients do not monitor their blood glucose levels as often as recommended and this presents a further barrier to appropriate insulin replacement and to the achievement of treatment goals.

Continuous glucose monitoring devices provide a complete picture of the $24 \mathrm{~h}$ glucose profile and should provide the information needed to achieve more physiological insulin replacement. These devices also reduce the need to perform frequent fingerstick tests.

In all of the glucose monitoring systems that are on the market, the glucose sensor does not directly measure blood glucose changes, but the sensor is inserted into the abdominal subcutaneous fat tissue to measure glucose changes in the interstitial fluid, or fluid is extracted transdermally or glucose is measured non-invasively. Subsequently, rapid changes in blood glucose must not result in respective changes in the signals measured by the glucose sensors due to their remote location. However, our knowledge about the time lag associated with such changes is very limited.

The first continuous glucose monitoring system (CGMS, Medtronic MiniMed, Northridge, CA, USA) has been marketed for several years; however, this system only provides retrospective data. Other devices are now available (GlucoWatch, Cygnus; GlucoDay, Menarini Diagnostics, Florence, Italy), or will soon reach the market (Pendra, Pendragon Medical, Zurich, Switzerland), which provide real time data presentation. In the future, automatic control of blood glucose levels will be possible using 'closed-loop systems' that are comprised of a glucose sensor and an insulin pump. However, significant hurdles (i.e. reliable performance of the glucose sensors, development of algorithms that allow coverage of prandial insulin requirements, rapidity of insulin adjustment) must be overcome before such systems become a clinical reality.

\section{Novel antihyperglycaemic agents}

A number of novel therapeutic agents have been developed or are under development for the treatment of type 2 diabetes, including insulin sensitisers (e.g. dual agonists of peroxisome proliferator-activated receptor 
(PPAR) $\alpha$ and PPAR $\gamma$ ), glucagon-like peptide-1 (GLP-1) analogues and derivatives (e.g. exendin-4), beta-3 receptor agonists and potassium channel openers. Currently available therapeutic agents have a number of substantial limitations, the most important of which is their inability to prevent disease progression owing to declining beta-cell function and their subsequent lack of long-term efficacy.

The intestinal peptide hormone GLP-1 has received much interest because of its potential to treat type 2 diabetes. Subcutaneous injection of GLP-1 increases insulin secretion, decreases glucagon secretion and delays gastric emptying - all of which contribute to lower blood glucose levels. However, GLP-1 has proven difficult to use therapeutically because it is rapidly inactivated by the enzyme dipeptidyl peptidase IV (DPP-IV). This may be overcome by inhibiting DPP-IV or using GLP-1 analogues that are resistant to DPP-IV degradation. In a Phase II study of patients with type 2 diabetes, once daily subcutaneous administration of the DPP-IV-resistant GLP-1 analogue LY307161 SR resulted in significant, dose-dependent decreases in blood glucose levels after 4 weeks (15). Thus, agents that utilise the insulinotropic properties of GLP-1 may be viable treatment options for patients with type 2 diabetes in the future.

The use of potassium channel openers is one strategy currently being investigated to reduce the beta-cell workload. It is postulated that resting beta-cells will result in restoration of beta-cell secretion, reduction in insulin resistance and prevention of beta-cell degeneration. However, this remains to be confirmed in human studies. Beta-3 receptor agonists are effective insulin sensitising agents in rodents and their potential to reduce blood glucose levels in humans is being tested. Activation of beta-3 adrenoceptors stimulates fat oxidation, thereby lowering intracellular concentrations of metabolites, e.g. fatty acyl CoA and diacylglycerol, which modulate insulin signalling. To date, however, difficulties in developing a compound with suitable efficacy, selectivity and pharmacokinetic properties have prevented any beta- 3 receptor agonist from progressing beyond early clinical trials (16).

\section{Conclusions}

A variety of new diabetes therapies and technologies are being developed. Critical evaluation of the therapeutic benefits will be mandatory before these novel treatment options become available for clinical use. The efficacy of antidiabetic agents is often lower in clinical practice than in controlled clinical trials owing to poor compliance and other problems that prevent optimal diabetes care. New therapeutic agents should ideally offer improved flexibility and tolerability over current therapeutic options in order to increase acceptability of therapy, with an equivalent or improved glucose lowering effect. Cost-benefit issues must also be considered, particularly in light of the growing financial burden of diabetes. The premium costs of new therapeutic agents and glucose sensors should be considered in the context of the potential optimisation of metabolic control, avoidance of hypoglycaemic events and the prevention of long-term complications.

The availability of new diabetes management options will help treatment strategies to be tailored to the needs of individual patients thereby helping them to achieve the best possible metabolic control. They will also provide physicians with the appropriate tools to overcome the obstacles to improve metabolic control and subsequently improve diabetes outcomes.

\section{References}

1 Heinemann L. Hypoglycemia and insulin analogues: Is there a reduction in incidence? Journal of Diabetes and its Complications 199913 105-114.

2 Heise T \& Heinemann L. Rapid and long-acting analogues as an approach to improve insulin therapy: an evidence-based medicine assessment. Current Pharmaceutical Design 20017 1303-1325.

3 Heinemann L, Linkeschova R, Rave K, Hompesch B, Sedlak M \& Heise T. Time-action profile of the long-acting insulin analog insulin glargine (HOE901) in comparison with those of NPH insulin and placebo. Diabetes Care 200023 644-649.

4 Ratner RE, Hirsch IB, Neifing JL, Garg SK, Mecca TE \& Wilson CA. Less hypoglycemia with insulin glargine in intensive insulin therapy for type 1 diabetes. U.S. Study Group of Insulin Glargine in Type 1 Diabetes. Diabetes Care 200023 639-643.

5 Yki-Jarvinen H, Dressler A \& Ziemen M. HOE 901/300s Study Group. Less nocturnal hypoglycemia and better post-dinner glucose control with bedtime insulin glargine compared with bedtime NPH insulin during insulin combination therapy in type 2 diabetes. HOE 901/3002 Study Group. Diabetes Care 200023 1130-1136.

6 Hermansen K, Madsbad S, Perrild H, Kristensen A \& Axelsen M. Comparison of the soluble basal insulin analog insulin detemir with NPH insulin: a randomized open crossover trial in type 1 diabetic subjects on basal-bolus therapy. Diabetes Care 200124 296-301.

7 Rosenstock J, Schwartz SL, Clark CM Jr, Park GD, Donley DW \& Edwards MB. Basal insulin therapy in type 2 diabetes: 28-week comparison of insulin glargine (HOE 901) and NPH insulin. Diabetes Care 200124 631-636.

8 Vague P, Selam JL, Skeie S, De Leeuw I, Elte JW, Haahr H, Kristensen A \& Draeger E. Insulin detemir is associated with more predictable glycemic control and reduced risk of hypoglycemia than NPH insulin in patients with type 1 diabetes on a basal-bolus regimen with premeal insulin aspart. Diabetes Care $200326590-596$.

9 Home PD, Boulton AJM, Jimenez J, Landgraf R, Osterbrink B \& Christiansen JS on behalf of the Worldwide Initiative for Diabetes Education (WorldWIDE). Issues relating to the early or earlier use of insulin in type 2 diabetes. Practical Diabetes International 2003 $2063-71$.

10 Pfützner A, Heise T, Steiner S, Heinemann L \& Rave K. Inhaled Technosphere/Insulin shows a low variability in metabolic action in type 2 diabetic patients. Diabetes 200049 (Suppl 1) A121.

11 Heise T, Rave K, Bott S, Sha S, Willavize SA \& Carroll RS. Timeaction profile of an inhaled insulin preparation in comparison to insulin Lispro and regular insulin. Diabetes 200049 (Suppl 1) A10.

12 Gerber RA, Cappelleri JC, Kourides IA \& Gelfand RA. Treatment satisfaction with inhaled insulin in patients with type 1 diabetes: a randomized controlled trial. Diabetes Care 200124 1556-1559. 
13 Cappelleri JC, Cefalu WT, Rosenstock J, Kourides IA \& Gerber RA. Treatment satisfaction in type 2 diabetes: a comparison between an inhaled insulin regimen and a subcutaneous insulin regimen. Clinical Therapeutics $2002 \mathbf{2 4} 552-564$.

14 Rosenstock J, Cappelleri JC, Nadkarni S, Petrie CD \& Gerber RA. Long-term Treatment Satisfaction with Inhaled Insulin (Exubera) and Improved Glycemic Control in Patients with Type 1 and Type 2 Diabetes. American Association of Clinical Endocrinologists 2003 Abstract.

15 Trautmann ME, Chen CF, Chappell JC, Dananberg J, Patterson B, Klausmann G, Kapitza C \& the GFFH Study Group. LY307161 SR, a long-acting GLP-1 analog, improved glycemic control in patients with Type 2 diabetes. American Diabetes Association 62nd Annual Scientific Sessions 2003. Abstract 582-p.

16 Arch JR. Beta(3)-Adrenoceptor agonists: potential, pitfalls and progress. European Journal of Pharmacology $20024 \mathbf{4 4 0} 99-107$.

Received 19 May 2004

Accepted 24 May 2004 